\title{
Computationally Efficient Technique for Nonlinear Poisson-Boltzmann Equation
}

\author{
Sanjay Kumar Khattri \\ Department of Mathematics, University of Bergen, Norway \\ sanjay@mi.uib.no \\ http://www.mi.uib.no/ sanjay
}

\begin{abstract}
Discretization of non-linear Poisson-Boltzmann Equation equations results in a system of non-linear equations with symmetric Jacobian. The Newton algorithm is the most useful tool for solving nonlinear equations. It consists of solving a series of linear system of equations (Jacobian system). In this article, we adaptively define the tolerance of the Jacobian systems. Numerical experiment shows that compared to the traditional method our approach can save a substantial amount of computational work. The presented algorithm can be easily incorporated in existing simulators.
\end{abstract}

\section{Introduction}

Lets consider the following non-linear elliptic problem

$$
-\operatorname{div}(\epsilon \operatorname{grad} p)+f(p, x, y)=b(x, y) \quad \text { in } \quad \Omega \quad \text { and } \quad p(x, y)=p^{D} \quad \text { on } \quad \partial \Omega_{D} .
$$

The above problem is the Poisson-Boltzmann equation arising in molecular biophysics. See the References [2, 7, 9, 10, 11, 12. Here, $\Omega$ is a polyhedral domain in $\mathbb{R}^{2}$, the source function $b$ is assumed to be in $L^{2}(\Omega)$ and the medium property $\epsilon$ is uniformly positive.

A Finite Volume discretization of the nonlinear elliptic equation results in a system of non-linear equations

$$
\mathbf{F}(\mathbf{p}):=\mathbf{A}_{1} \mathbf{p}_{h}+\mathbf{A}_{2}\left(\mathbf{p}_{h}\right)-\mathbf{b}_{h}=0
$$

Here, $\mathbf{F}=\left[F_{1}(\mathbf{p}), F_{2}(\mathbf{p}), \cdots, F_{n}(\mathbf{p})\right]^{T}, \mathbf{A}_{1}$ is the discrete representation of the symmetric continuous operator $-\operatorname{div}(\epsilon \operatorname{grad})$ and $\mathbf{A}_{2}$ is the discrete representation of the non-linear operator $f(p, x, y)$.

A Newton-Krylov method for solving the non-linear equation (2) is given by the Algorithm 1, In the Quasi-Newton method (see Algorithm 2), we are solving the Jacobian equation $\left(\boldsymbol{J}\left(\mathbf{p}_{k}\right) \Delta \mathbf{p}=-\mathbf{F}\left(\mathbf{p}_{\mathbf{k}}\right)\right)$ approximately. We are solving the system $\boldsymbol{J}\left(\mathbf{p}_{k}\right) \Delta \mathbf{p}_{k}=-\mathbf{F}\left(\mathbf{p}_{k}\right)+\mathbf{r}_{k}$ with $\left\|\mathbf{r}_{k}\right\|$ is chosen adaptively. The quasiNewton iteration is given by the Algorithm 2, In the Algorithms 1 and 2, $\|\cdot\|_{L_{2}}$ denotes the discrete $L_{2}$ norm and $\max _{i t e r}$ is the maximum allowed Newton's iterations. It is interesting to note the stopping criteria in the Algorithm 2. We 

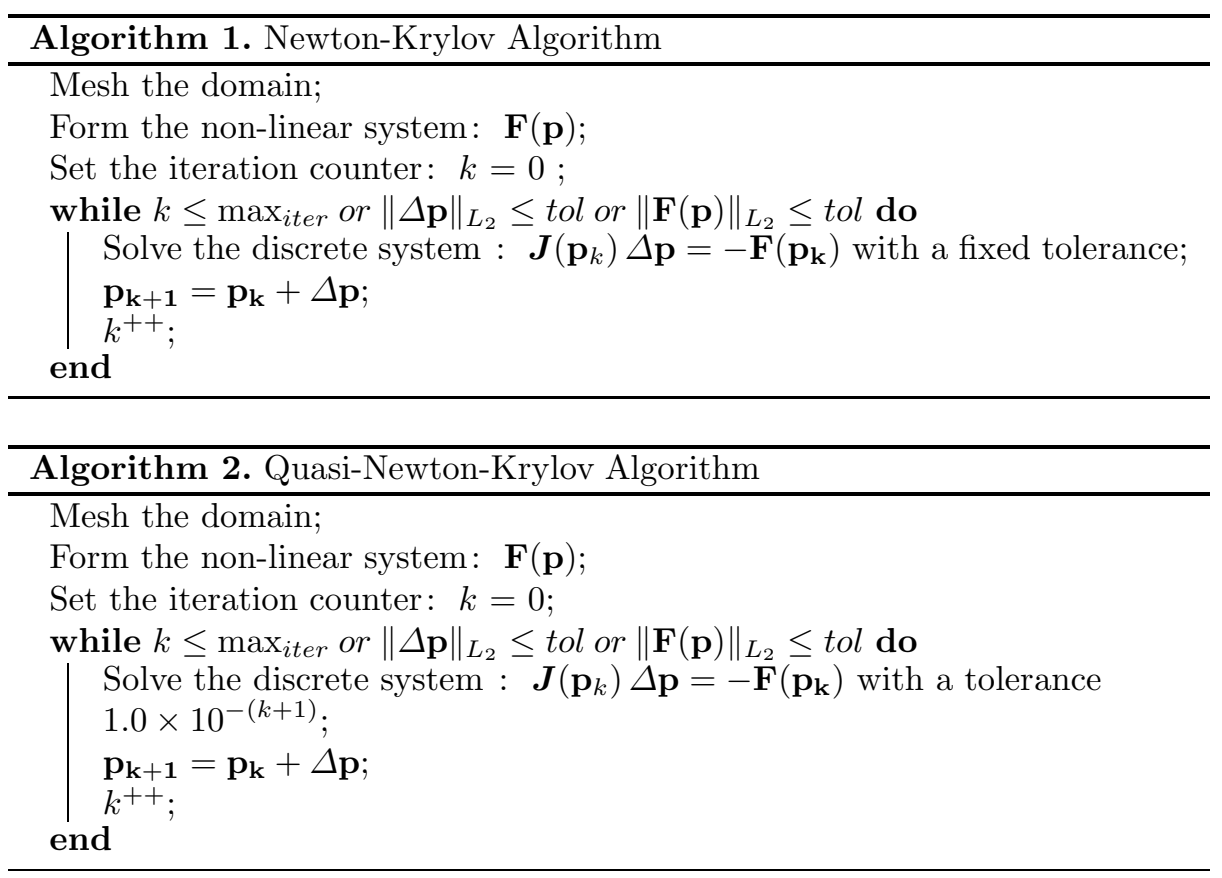

are using three stopping criterion in the Algorithms. Apart from the maximum allowed iterations, $L_{2}$ norm of residual vector $\left(\|\mathbf{F}(\mathbf{p})\|_{L_{2}}\right)$ and also $L_{2}$ norm of difference in scalar potential vector $\left(\|\Delta \mathbf{p}\|_{L_{2}}\right)$ are being used as stopping criterion for the Algorithms. Generally in the literature, maximum allowed iterations and the residual vector are used as stopping criteria [9, 10, 11, and references therein]. If the Jacobian is singular than the residual vector alone cannot provide a robust stopping criteria.

\section{Numerical Experiment}

Let us solve (3) in the domain $\Omega=[-1,1] \times[-1,1]$ with $k=1.0$ [2, 7, 8, 9]. $\Omega$ is divided into four equal sub-domains (see Figure 1) based on $\epsilon$.

$$
-\nabla \cdot(\epsilon \nabla p)+k \sinh (p)=f \quad \text { in } \quad \Omega \quad \text { and } \quad p(x, y)=x^{3}+y^{3} \quad \text { on } \quad \partial \Omega_{D} .
$$

For solving the linear systems, we are using ILU-preconditioned the ConjugateGradient (CG) method. For the Newton algorithm the tolerance of the CG method is $1.0 \times 10^{-15}$. For the quasi-Newton method the tolerance of the CG method varies with the iterations $k$ of the Algorithm 2 as follows : $1.0 \times 10^{-(k+1)}$, $k=0,2, \ldots, 14$. Figures 3 , 4 and 2 reports the outcome of our numerical work. The Figures 3 and 4 compares convergence of the quasi-Newton and Newton methods. The Figure 2 reports computational complexity of the quasi-Newton 


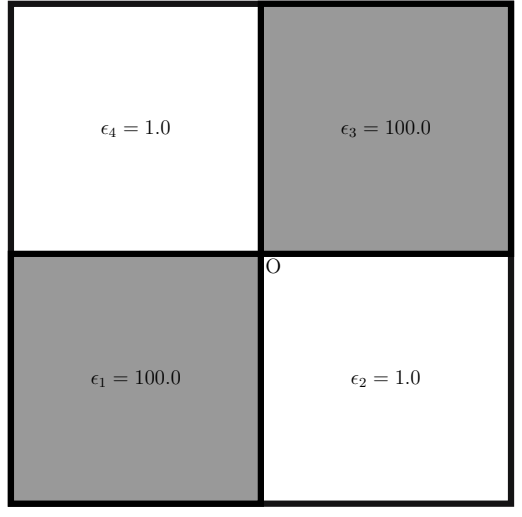

Fig. 1. Distribution of medium property $\epsilon$ in the domain $\Omega=[-1,1] \times[-1,1]$

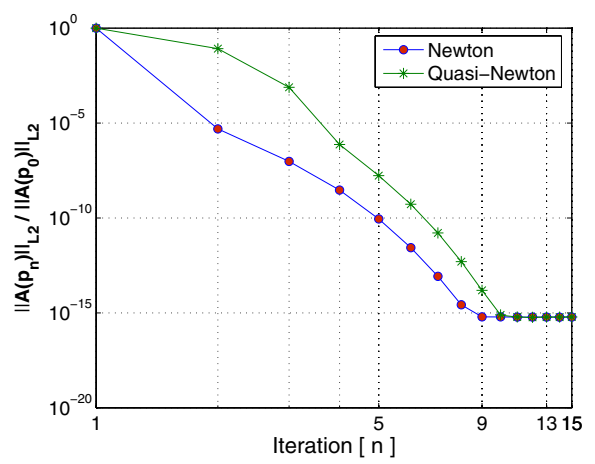

Fig. 3. Convergence of the $L_{2}$ norm of residual vector $\mathbf{A}(\mathbf{p})$

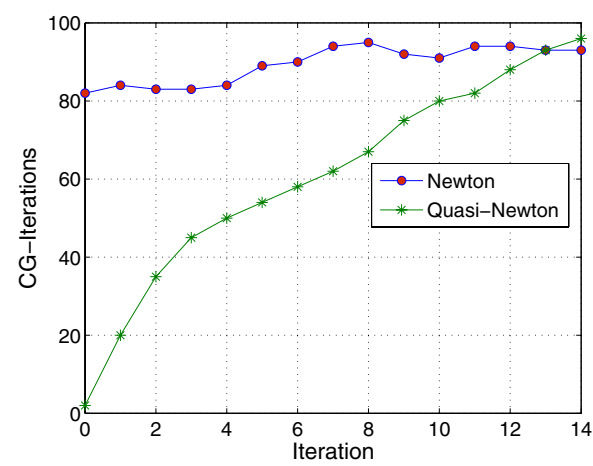

Fig. 2. Computational work required by the Quasi-Newton and Newton methods

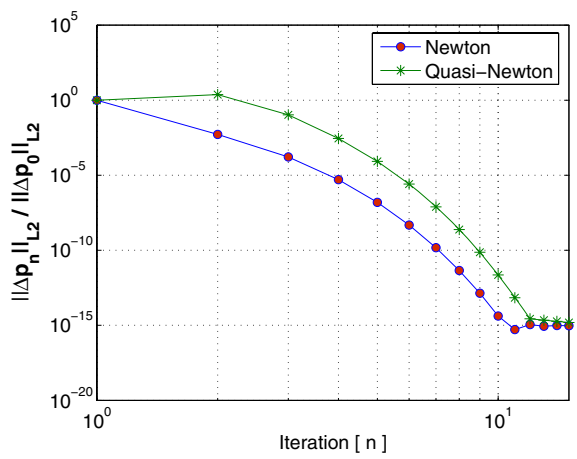

Fig. 4. Convergence of the $L_{2}$ norm of difference vector $\Delta \mathbf{p}$

and the Newton methods. It can be notice, even if initial iterations of the NewtonKrylov algorithm are solved approximately, the convergence rate of the algorithm remains unaffected. The Figure 2 shows that such an approximation saves a substantial amount of computational effort.

\section{Conclusions}

Quasi-Newton method for solving non-linear system of equation with symmetric Jacobian matrix is presented. Numerical work shows that the presented technique is computationally efficient compared to the traditional Newton-Krylov method. An efficient solution technique for Poisson-Boltzmann equation is of interest to the researchers in computational chemistry, bio-physics and molecular dynamics. The presented algorithm can be easily implemented in existing simulators. 


\section{References}

1. Khattri, S.K.: Analyzing Finite Volume for Single Phase Flow in Porous Media. Journal of Porous Media. Accepted for Publication, (2006).

2. Aksoyw, B.: Adaptive Multilevel Numerical Methods with Applications in Diffusive Biomolecular Reactions. PhD Thesis, The University of California, San Diego (2001).

3. Khattri, S.K.: Newton-Krylov Algorithm with Adaptive Error Correction for the Poisson-Boltzmann Equation. MATCH Commun. Math. Comput. Chem., 56, (2006).

4. Chow, S.-S.: Finite element error estimates for nonlinear elliptic equations of monotone type. Numer. Math., 54, (1989), 373-393.

5. Eymard, R., Gallouët, T., Hilhorst, D. and Naït Slimane, Y.: Finite volumes and nonlinear diffusion equations. RAIRO Math. Model. Numer. Anal., 32, (1998), $747-761$.

6. Lui, S.H.: On Schwarz Alternating Methods For Non Linear Elliptic PDEs. SIAM Journal on Scientific Computing, 21, (2000), 1506-1523.

7. Fogolari, F., Brigo, A. and Molinari, H.: The Poisson Boltzmann equation for Biomolecular electrostatics: A Tool for Structural Biology. Journal of Molecular Recognition, John Wiley \& Sons Ltd., 15, (2002), 377-392.

8. Kuo, S.S., Altman, M.D., Bardhan, J.P., Tidor, B. and White, J.K. : Fast Methods for Simulation of Biomolecule Electrostatics. International Conference on Computer Aided Design, (2002).

9. Host, M., Kozack, R.E., Saied, F. and Subramaniam, S.: Treatment of Electrostatic Effects in Proteins: Multigrid-based Newton Iterative Method for Solution of the Full Nonlinear Poisson-Boltzmann Equation. Proteins: Structure, Function, and Genetics, 18, (1994), 231-245.

10. Host, M., Kozack, R.E., Saied, F. and Subramaniam, S.: Protein electrostatics: Rapid multigrid-based Newton algorithm for solution of the full nonlinear PoissonBoltzmann equation. J. of Biomol. Struct. \& Dyn., 11, (1994), 1437-1445.

11. Host, M., Kozack, R.E., Saied, F. and Subramaniam, S.: Multigrid-based Newton iterative method for solving the full Nonlinear Poisson-Boltzmann equation. Biophysical Journal, 66, (1994), A130-A130.

12. Holst, M. and Saied, F.: Numerical solution of the nonlinear Poisson-Boltzmann equation: Developing more robust and efficient methods. J. Comput. Chem., 16, (1995), 337-364.

13. Baker, N., Sept, D., Holst, M. and McCammon, J.A.: The adaptive multilevel finite element solution of the Poisson-Boltzmann equation on massively parallel computers. IBM J. Research and Development, 45, (2001), 427-438. 